\title{
DIFFERENTIAL ANALYSIS OF SOFC CURRENT-VOLTAGE CHARACTERISTCS
}

\author{
Zdravko Stoynov ${ }^{1}$, Daria Vladikova ${ }^{1}{ }^{*}$, , Blagoy Burdin ${ }^{1}$, Jerome Laurencin ${ }^{2}$, Dario \\ Montinaro $^{3}$, Gergana Raikova ${ }^{1}$, Günter Schiller ${ }^{4}$, Patric Szabo ${ }^{4}$ \\ ${ }^{1}$ Institute of Electrochemistry and Energy systems - BAS, 10 Acad. G. Bonchev St., 1113 \\ Sofia, Bulgaria \\ ${ }^{2}$ Univ. Grenoble Alpes - CEA/LITEN, 17 rue des Martyrs, 38054 Grenoble, France \\ ${ }^{3}$ SOLIDpower S.p.A, Viale Trento 117, 38017 Mezzolombardo, Italy \\ ${ }^{4}$ German Aerospace Center (DLR), Linder Höhe, 51147 Cologne, Germany
}

\begin{abstract}
Solid Oxide Fuel Cells (SOFC) are regarded as a promising technology for economic power generation due to their high efficiency and large fuel flexibility. Durability is a severe hurdle towards their deployment. The near future targets in respect to Degradation Rate (DR) are about $0.1 \%$.kh-1, which needs improved monitoring and diagnostics. This work aims at introducing new approach based on Differential Analysis of the i-V curves, named DiVA. It operates with the Differential Resistance (DR) $R$ d and its evolution during long term testing. Since derivatives are more sensitive to small deviations, the Differential Resistance Analysis (DRA) ensures increased sensitivity and information capability in respect to degradation monitoring and diagnostics, which is demonstrated on a small stack during thermal cycling conditions - before and after the first thermal cycle, on button cells tested up to 9000 hours, as well as on button cells operating in fuel cell and in electrolysis mode. The results show that DRA is several times more sensitive in comparison with the classical DR evaluation based on registration of the voltage decrease at constant current.
\end{abstract}

Key word: current-voltage characteristics; degradation rate, differential resistance analysis, durability 


\section{INTRODUCTION}

Solid Oxide Fuel Cells (SOFCs) appear as an attractive technology for economic power generation, presenting high efficiency and large fuel flexibility. The key challenge is to achieve a competitive balance between costs, efficiency and durability - three leading parameters that are internally linked and mutually optimized following definite key performance indicators [1]. In respect to durability the near future targets for stationary applications are $80000 \mathrm{~h}$ life-time. For improvement and optimization of SOFC long term performance it is important to identify and quantify the degradation sources and to use them as a base for the development of successful mitigation strategies.

The most commonly applied electrochemical test for quantitative evaluation of SOFC performance is the long term stable operation at a given load which is presented with the V$\mathrm{t}$ (voltage- time) characteristic and the periodically measured instantaneous discharge $\mathrm{i}-\mathrm{V}$ (current voltage) curves which give integral picture of the system (cell, stack) for a definite state. Very often for separation of the degradation sources impedance measurements are also performed. However, they describe single working points selected from the i-V curves.

For evaluation of the degradation process the parameter "degradation rate" (DR) is introduced. Usually the decrease of the voltage for 1000 hours operation (mV.kh-1) at constant load is used. A linearized average degradation rate for the whole testing time, or segmented one, defining different degradation stages as initial and long term degradation, are applied. The voltage change may be replaced with that of the corresponding area specific resistance $\left(\mathrm{m} \Omega . \mathrm{cm}_{2} . \mathrm{kh}-1\right)$ [2,3]. For convenience the DR is expressed also in \%. The degradation rate currently reported for SOFCs is below 1\%.kh-1, while the near future target is 10 times less, i.e. $0.1 \%$.kh-1 [3-5]. For Solid Oxide Electrolyzers (SOEL) it exceeds $3 \%$.kh-1 [6].

The precise calculation of the DR already needs long tests (several years) which are neither appropriate as information source for further optimization, nor convenient for implementation. The problem solving approach, which is under active development, is the introduction of accelerated stress tests (AST) and sophisticated performance/degradation models to quantify the accelerating impact. They should shorten testing time, however, the experimental condition should activate the same ageing mechanisms as in non-accelerated testing, thus preventing the system from eventual irreversible changes which may bring to 
false results. The selection of the acceleration conditions is a critical moment in the procedure. Since there are no definite criteria for regulation the level of acceleration, less severe conditions are preferable. Thus to obtain the desired decrease of the testing time in "safe acceleration mode" and to detect and identify first signals of critical operation, improved monitoring with increased sensitivity is also needed.

During electrochemical testing a big experimentally linked data set is accumulated. It can be further exploited. Current-voltage characteristics are the simplest external general description of the electrochemical power source ability to produce electrical energy. They describe the interrelation between the power components - voltage and current, covering the entire power source operating region. As far as electrochemical reactions are non-linear in nature, in general iV curves are also non-linear. As an external description they reflect the dominant phenomena governing the process of electrical energy generation, i.e. they represent the dominant hindrances. The typical fuel cell $\mathrm{i}-\mathrm{V}$ characteristic is a decreasing curve (Fig.1), characterized with three main regions. At low currents a fast decreasing pseudo-exponential first region is observed (Segment I in Fig. 1), where the main factor contributing to the nonlinear voltage drop is the activation of the electrochemical reactions. As the current increases, the curve becomes close to linear for a large range of currents. (Segment II in Fig. 1). It is associated with the ionic and electronic transport - mainly in the electrolyte, but also towards the interconnects. Those processes are in general linear and they constitute the linear components of the $\mathrm{i}-\mathrm{V}$ curve. At high currents the curve becomes again non-linear (Segment III in Fig. 1) and steep due to the concentration (mass transport) voltage drop caused by the fast consumption of the reactive gases $[7,8]$.

Fig. 1.

The produced power output $P$ versus current (Fig. 1) has a specific shape: the power increases continuously with the increase of the load current and attains its maximum value at certain load. The region of the maximum power $P_{\max }$ shapes a specific plateau, followed by a rapid fall due to the increase in the ohmic and concentration (mass transportation) losses. For every point of the $\mathrm{iV}$ characteristic the internal resistance $R_{\mathrm{i}}$ can be also calculated as a ratio of the voltage drop from OCV (open circuit voltage) and the correspondent current. When calculated for $P_{\max }$, it has a minimum value $R_{\mathrm{i}, \min }$ which can be also expressed as area specific resistance $\left(A S R_{\min }\right)$. The vector of the parameters extracted from the $\mathrm{i}-\mathrm{V}$ curves $\left(\mathrm{OCV}, P, P_{\max }, R_{\mathrm{i}, \min }\right.$, $\left.A S R_{\min }\right)$ which describes in the most general way the ability of a given power source to 
produce power, is used for characterization of fuel cells during their design, optimization and operation analysis. It forms the kernel of the Performance Vector, which contains also the operation conditions.

The external behavior represented by $\mathrm{i}-\mathrm{V}$ curves is an integral product of a series of processes taking place in the constructive elements of the fuel cell and at their interfaces, i.e. it reflects all dominant phenomena governing the process of electrical energy generation and thus - the dominant barriers. Multiple studies are devoted to the deeper insight in the nature of the elementary electrochemical steps. However, taking into account the different origin of the processes (part of them are in solid state, others - at triple phase boundaries, at the interface, in different gases environment etc.), the full deterministic description of the integral external behavior is rather difficult. As a consequence the $\mathrm{i}-\mathrm{V}$ curves should be discussed as an external exhibition of statistical (and dynamically stochastic) large system properties, conditioned in definite operating conditions. Those considerations suggest for application of statistical approaches for further analysis of the information enclosed in them. One effective direction is the analysis of the differential resistance. It has been suggested by some authors [2-4], but no sufficient progress is registered in the literature.

This work aims at introducing a new approach which applies Differential Analysis of the i-V characteristics, named DiVA. It operates with the differential (tangential) internal resistance $R \mathrm{~d}$ and its evolution during long term testing. The performed Differential Resistance Analysis (DRA) ensures increased sensitivity and information capability in respect to degradation monitoring and diagnostics which is shown in a number of examples on SOFC. They are performed for approbation of the method, for definition of its application niches and for framing future requirements and trends in its development

\section{RESULTS AND DISCUSSION}

\section{Principle of DiVA}

The current-voltage characteristics are sensitive to operating parameters and degradation which reflects in the change of their shape. Thus a quantitative estimation of the i-V shape and its change during operation at constant conditions can serve as performance indicator. The parameter that reflects the shape is the internal resistance, which takes different values in different working points (Fig. 1). The DiVA approach is based on the calculation of the 
Differential Resistance $R$ d, i.e. the derivative of the voltage $U$ in respect to the corresponding current $I$ :

$$
R_{\mathrm{d}}=\mathrm{d} U / \mathrm{d} I
$$

The values of $R \mathrm{~d}$ for every point of the $\mathrm{i}-\mathrm{V}$ curve constitute a new function:

$$
R_{\mathrm{d}}=\mathrm{f}(I)
$$

Thus the innovative "Shape analysis" of the $\mathrm{i}-\mathrm{V}$ curve is based on the analysis of this function, i.e. on the current dependence of $R \mathrm{~d}$, named Differential Resistance Analysis (DRA) (Fig. 2a). Being a function of a derivative, it is more sensitive to small deviations which apriori increases the sensibility towards degradation. In the general case of "s" type shape (resemblance with the letter "s"), the i-V curve and the derivative function (2) are non-linear (Fig.2a). The differential resistance sharply decreases in the beginning, i.e. at low current (Segment I), which is connected with the activation losses, followed by a relatively flat minimum (Segment II) corresponding to the transport hindrances and then it increases again (Segment III) due to domination of gas diffusion limitations. As a derivative function, it contains a lot of information which should be analyzed further.

\section{Fig. 2.}

An important characteristic point for the cell performance is the minimum of the differential resistance $R_{\mathrm{d}, \mathrm{min}}$. In general it reflects the state of health at constant operating conditions, since it is determined by the intrinsic properties of the system and not by the external conditions (for instance load current). The defined value of $R_{\mathrm{d} \text {,min }}$ corresponds to the zero point of the derivative $R_{\mathrm{d}}$, i.e. it can be regarded as a second derivative. As a result, changes of $R_{\mathrm{d} \text {,min }}$ caused by the state of the system become observable much earlier than those of other parameters selected for monitoring and evaluation of the state of health.

The DRA can be presented in the more illustrative spectral form (Fig. 2b). The spectral peak describes Segment II in Fig. 2a, while segments I and III are introduced in the spectral tail. The spectrum has 2 characteristic points: (i) $R_{\mathrm{d}, \min }$ - the minimum value of the differential resistance and (ii) $R_{\text {int, } \max }$ - the value of the spectral maximum, which gives the most stable value of $R$ d, i.e. the value which is observed in a wider current range. The intensity of the 
spectral pick is proportional to the current range with similar values of the differential resistance. The sharper the spectral line, the smaller the distribution of this parameter.

When the VAC is of "s"- type shape, as it is in Fig. 2a, the values of $R \mathrm{~d}$ from Segments I and III are overlapping in their spectral presentation. In order to avoid ambiguity, a separation can be performed by presenting the spectrum as "Twin Spectra" reflecting the behavior of the $R \mathrm{~d} / I$ dependence before and after $R_{\mathrm{d}, \mathrm{min}}$ (Fig. 2c).

The spectral transform is not simply a presentation of the same data in different coordinates. By its nature it is a type of regression analysis, filtrating the statistical noises coming from the object itself and from the performed measurements. This analytical tool has a high power of filtration, eliminating the possible wild points and the resulting "black" noise.

The DRA determines the value of $R_{\mathrm{d}, \min }$ and the corresponding current (Fig. 2d). The tangential in this point $\left(I_{\mathrm{Rd}, \min }\right)$ defines the voltage $\mathrm{U}_{00}$ which is the voltage at $I=0$ in case the system would work with resistance equal to $R$ d,min. The difference $\Delta U^{*}=U_{0}-U_{00}$ serves as a new indicator. Although partially connected with the position of $R_{\mathrm{d}, \mathrm{min}}$, it is more sensitive to the activation losses. Thus the $\mathrm{i}-\mathrm{V}$ shape analysis performed by the DRA introduces two additional performance indicators: $R_{\mathrm{d}, \min }$ and $\Delta U^{*}$.

For degradation analysis from the $\mathrm{i}-\mathrm{V}$ curves a combined exploration of the derived Vector of the parameters should be applied, since they may be sensitive to different degradation processes and sources. It should be pointed that $R_{\mathrm{d} \text {,min }}$ does not correspond to $R_{\mathrm{Pmax}}$.

The operation with derivatives increases the sensitivity of the produced results. However this is connected with increase of data noise which requires acquisition of more precise and confident data. DiVA needs higher quality of the measurements and not a big number of data. For one i-V curve 30-40 points are sufficient, however, they should be measured after stabilization of the operating conditions. The recording of the volt ampere characteristics is performed point by point - the current is swept step-wise and the corresponding voltage is measured. Between every sweep and measurement a specific delay time should be introduced - it ensures the relaxation of the dynamic transition processes and provides for a stationary measurement. In the case of SOFC the delay time is typically 20 to 30 seconds. More details 
about the recommendations for measurements when applying DiVA are given in [9].

\section{Examples on DiVA Applications}

The selected examples for DiVA assessment are taken from experiments on SOFC/SOEL degradation studies $[10,11]$. In general the tested cells/stacks are based on planar configuration with dense YSZ electrolyte with thickness of about 5-10 $\mu \mathrm{m}$ supported by a thick porous Ni-YSZ hydrogen electrode of $260 \mathrm{~mm}$. The oxygen electrode is a porous LSCF. A thin barrier layer of CGO is deposited between the electrolyte and the LSCF electrode for limitation of the chemical reactivity. A pure LSC layer is used for current collector.

\section{DiVA Sensitivity Assessment}

For assessment of DiVA sensitivity and applicability for early warning signals (EWOS), the method was applied on short 4 cells stack (active cell area $80 \mathrm{~cm}^{2}$ ) produced by SOLIDpower. The polarization curves were measured by DLR in SOFC stress test regime of thermal cycling under the following conditions: heating up to $750{ }^{\circ} \mathrm{C}$ with $2 \mathrm{k} / \mathrm{min} ; 2$ hours stabilization at OCV; i-V curve measurement with $U_{\min }=2.8 \mathrm{~V}$; cooling down to $100^{\circ} \mathrm{C}$ with $2 \mathrm{k} / \mathrm{min}$. The selected fuel gas flow was $1.15 \mathrm{Nl} / \mathrm{min} \mathrm{H}_{2}$ and $0.77 \mathrm{Nl} / \mathrm{min} \mathrm{N}_{2}$ while $21.3 \mathrm{Nl} / \mathrm{min}$ of air was used on the cathode side. Gas flows were kept constant in order to avoid any redox cycling of the anode. The stack was run for about $1700 \mathrm{~h}$ and was subject to 34 thermal cycles. The initial OCV was $4.78 \mathrm{~V}$ and the final $4,76 \mathrm{~V}$. The maximum power $P_{\max }\left(\right.$ at $\left.410 \mathrm{~mA} / \mathrm{cm}^{2}\right)$ of the pristine stack $(80 \% \mathrm{FU})$ was $323 \mathrm{~mW} / \mathrm{cm}^{2}$. It decreased down to $309 \mathrm{~mW} / \mathrm{cm}^{2}$ after 34 cycles. A degradation of $2.5 \% \cdot \mathrm{kh}^{-1}$, or $0.12 \% /$ thermal cycle was calculated.

Obviously it is difficult to evaluate accurately the DR from one, or even from several thermal cycles. Thus the sensitivity of the DiVA approach was checked for a single thermal cycle the first one. The testing was based on comparative evaluation of the change (expressed in \%) of $R_{\mathrm{d}, \min }, \Delta U^{*}$ and $P_{\max }$, the last one taken as a mean value from the long term cycling. As seen in Fig. 3a, no observable difference in the i-V curves before and after the first thermal cycle can be registered, as well as in $P_{\max }$ at $410 \mathrm{~mA} / \mathrm{cm}^{2}$, while the changes in the performance indicators determined by DRA are more than $7 \%$ (Fig. 3 b,c).

Fig. 3. 
Since the increase of $\Delta U^{*}$ and $R_{\mathrm{d}, \min }$ can be attributed to dominant enhancement respectively of the activation and transport hindrances, it may be supposed that the thermal cycling affects both of them. The results in Fig. 3c illustrate the higher sensitivity of the DRA which registers changes still undistinguishable directly from the i-V curves.

\section{DiVA Assessment for Degradation Monitoring}

The sensitivity of DiVA towards DR monitoring was approbated in long term (1000 to 9000h) degradation tests of button cells produced by SOLIDpower and tested in CEA with a composite LSCF/CGO oxygen electrode. Three testing regimes were selected, one of them in electrolysis mode (Table 1). It should be marked that the selected cells exhibit quite good performance. Their linearized average degradation rate calculated as the change of the voltage for 1000 hours operation at current load $0.5 \mathrm{Acm}^{2}$ (Table 1) ranges in the lower bounds of typical values reported for both fuel cell (FC) and electrolysis (EL) modes [12-18].

For the long term durability test (cell C1) two degradation stages were registered - initial one during the first 2000 hours with DR 13,5 mV.kh${ }^{-1}(1.64 \%)$ and a long term one lasting till the end of the experiment with about 3 times lower degradation (Table 1). The evaluation of the DRA for degradation monitoring followed the same approach, however, based on the timedependence of the performance indicators $R_{\mathrm{d}, \min }$ and $\Delta U^{*}$ extracted from the $\mathrm{i}-\mathrm{V}$ curves. More detailed analysis about the first 4000 is missing due to the lack of $\mathrm{i}-\mathrm{V}$ curves for the testing range 1000 - 3000 hours. The results from the DRA are presented in Table 1 and Fig. 4. While the $\mathrm{i}-\mathrm{V}$ curves give a qualitative picture of the degradation, illustrating small differences in the curves position and shape after 5000 hours (Fig. 4a), the time dependence of the performance indicators $R_{\mathrm{d}, \min }$ and $\Delta U^{*}$ ensures quantitative evaluation (Fig. 4b) which after linearization is presented as DR (Table 1). The results confirm the high sensitivity of the DRA. The period of ageing provokes a progressive increase of $R_{\mathrm{d}, \min }$ and $\Delta U^{*}$. For 9000 hours their change is about $25-30 \%$ compared to less than $8 \%$ for the voltage drop at $0,5 \mathrm{~A} / \mathrm{cm}^{2}$. The DRA distinguishes two degradation rates. The faster one is registered up to 5000 hours operation. In this range the increase of the performance indicators $R_{\mathrm{d}, \min }$ and $\Delta U^{*}$ is respectively 22 and $28 \%$, i.e. the DR is about $5 \% \cdot \mathrm{kh}^{-1}$, while for the last 4000 testing hours the DR decreases to $1,3 \% \cdot \mathrm{kh}^{-1}$ (Table 1$)$.

Fig. 4. 
Since the increase of $\Delta U^{*}$ and $R_{\mathrm{d} \text {,min }}$ can be attributed respectively to dominating activation and transport hindrances, it may be supposed that the long term performance affects both of them, which is, however, strongly pronounced in the first 5000 hours. This result is confirmed by the impedance measurements carried out at working point of $0,5 \mathrm{~A} / \mathrm{cm}^{2}$ (Fig. $4 \mathrm{c}$ ) which give a more detailed internal picture. It should be noted that this working point is not far away from the one corresponding to $R_{\mathrm{d} \text {,min. }}$. The diagrams show that the increase of the impedance in the first 5000 hours is determined by the: (i) ohmic resistance, i.e. by transport limitations and by the (ii) polarization resistance in its high frequency part, related to increase of the charge transfer resistance, i.e. by activation losses. Above 5000 hours testing the diagrams are identical in both position and shape. The detailed impedance analysis is not in the scope of this paper. The obtained results confirm the high sensitivity of the DRA which opens a new approach for degradation studies.

\section{DiVA Assessment in SOFC-SOEL Mode}

Solid Oxide Cells (SOC) are intrinsically reversible. They can be operated in electrolysis mode to produce hydrogen from steam, or in fuel cell (FC) mode to produce electricity. Using two separate devices increases the investment cost while higher efficiency and flexibility can be achieved if a single device can operate in both modes. Although from construction point of view SOELs and SOFCs look similar, there are big differences in the operating conditions which bring to high asymmetry. For instance the high current density associated with a large amount of water in the fuel electrode for SOEL compared to SOFC strongly increases the polarization resistance in that mode of operation [6]. The present SoA shows that in electrolysis mode the degradation is much higher - about $2-5 \%$ after $1000 \mathrm{~h}$ of operation, which is behind the commercialization thresholds [6]. In this respect special measures are taken for improvement of the electrodes performance and stability. The optimization of one material for two performance functions requires unified approach for monitoring and diagnostic of degradation during testing. Although the i-V curves testing is working for operation in both FC and in EL mode, the interpretation is not straightforward.

With regard to DiVA, the new performance indicators based on the DRA reflect the internal state of the system at definite operation conditions which makes $R_{\mathrm{d}, \min }$ and $\Delta U^{*}$ comparable for DR evaluation of a system operating in both FC and El mode. For confirmation of this statement the DRA was performed on $\mathrm{i}-\mathrm{V}$ characteristics for sells C2 and C3 [12] which were tested respectively in FC and EL mode for 1000 hours in equivalent operation conditions (Fig. 
5a, Table 1). The degradation rate extracted from the time-dependence of the voltage at constant current density $\left( \pm 0,5 \mathrm{~A} \cdot \mathrm{cm}^{-2}\right)$ was found to be about two times higher under electrolysis current, which indicates that the cell material ageing is accelerated under electrolysis polarization (Table 1). The results from the DRA confirm this difference, however, the registered changes in $R_{\mathrm{d}, \mathrm{min}}$ are more pronounced (Table 1) which again illustrates the higher selectivity of the DiVA approach. No changes in $\Delta U^{*}$ were observed, which is logical taking under consideration the linear behavior of the $\mathrm{i}-\mathrm{V}$ curves at low current (Fig. 5). Obviously the presence of water in the Ni/YSZ electrode in FC and EL mode decreases the activation losses.

Fig.5.

\section{CONCLUSIONS}

The results from this work confirm that DiVA approach based on the Differential Resistance Analysis is an useful tool with increased sensibility towards degradation, since it works with the derivatives of the measured parameters, which are in principle more sensitive to small deviations. It gives an opportunity for collection of reliable data from shorter tests avoiding severe accelerating test conditions. Since the DRA performance indicators are more sensitive, sharp deviations in their time dependence can serve as EWOS for increased degradation. Every indicator has higher selectivity in respect to different degradation sources: $\Delta U^{*}$ is more sensitive to activation losses and $R_{\mathrm{d} \text {,min }}$ - to transport hindrances. In addition impedance measurements can give more precise information about the origin of the degradation (ohmic losses, electrodes polarization etc.).

The practice of WOS (Warning Output Signals) application is largely used in other technical fields (machineries, buildings etc.). For SOFCs, however, the commonly accepted practice of life testing is based mainly on a simple measurement of the voltage at a constant current under constant conditioning. The produced results are voltage/time curves, which cannot ensure enough information for early diagnostic. An advancement in this direction is the applied periodic application of additional active input signals ( $\mathrm{i}-\mathrm{V}$ curves or impedance) for acquiring more informative outputs necessary for diagnostic purposes.

The development of the Differential Resistance Analysis provides for the introduction of EWOS technique for fuel cells. The defined value of $R_{\mathrm{d} \text {,min }}$ corresponds to the zero point of 
the $R_{\mathrm{d}}$ derivative, i.e. it can be regarded as a second derivative. Thus the changes of $R_{\mathrm{d} \text {,min }}$ can be registered earlier than those of other FC parameters.

The simplest EWOS approach comprises: (i) measurement of i-V characteristics - up to $P_{\max }$ (or another limiting parameter as $I_{\max }$, or $U_{\min }$ ); (ii) Differential Analysis - calculation of $R_{\mathrm{d}}$, $R_{\mathrm{d} \text {,min }}$ and $\Delta U^{*}$, Spectral Transform; (iii) consecutive performance of these two steps during the life testing at every $500 \mathrm{~h}$ (up to $2000 \mathrm{~h}$ ) and at every $1000 \mathrm{~h}$ after this limit; (iv) calculation of the relative deviations of $R_{\mathrm{d}, \min }$ and $\Delta U^{*}$ in respect to the pristine cell and as DR.

This procedure was successfully approbated with data of different origin, measured in different laboratories. All tests demonstrated applicability and efficiency of the described procedure. However, since they were initially performed for other targets, they did not fill DiVA requirements - usually for one i-V curve more than 2000 measurements were carried out without delay and accusation time, which reflected on the quality of the derivatives data. The application of the home made software product AWACS (Auto-Weighting Approximating Cubic Splines) and the spectral transform improved the noise immunity. However, for future applications the recommendation for $\mathrm{i}-\mathrm{V}$ curves comprising 30-40 points measured after stabilization of the operating conditions (about 20-40 seconds) is highly encouraged [9].

\section{ACKNOWLEDGMENT}

The research has received funding from FP7/2007-2013 Fuel Cells and Hydrogen Joint Undertaking (FCU-JU-2013-1) under grant agreement No 621207.

\section{REFERENCES}

[1] Fuel Cells and Hydrogen Joint Undertaking, Multi-Annual Work Plan 2014-2020, p.35, http://www.fch.europa.eu/sites/default/files/documents/FCH2\%20JU\%20\%20Multi\%20Annual\%20Work\%20Plan\%20-\%20MAWP_en_0.pdf

[2] N. Akkinapragada and B. H. Chowdhury, "SOFC-Based Fuel Cells for Load Following Stationary Applications," Proceedings of the 38th North American Power 
Symposium, 2006, Institute of Electrical and Electronics Engineers (IEEE), Jan 2007; http://dx.doi.org/10.1109/NAPS.2006.359626

[3] J. Hjelm in "Degradation testing - Quantification and Interpretation", 2nd International Workshop on degradation Issues of Fuel Cells, Thessaloniki, GR, 2011.

[4] R.S. Gemmen, M.C. Williams and K.Gerdes, J. Power Sources 184(1), 251-259 (2008).

[5] A. Hagen, R. Barfod, P. V. Hendriksen, Y.-L. Liu and S. Ramoussep J. Electrochem. Soc. 153(6) (2006) А1165-А1171л

[6] Fuel Cells and Hydrogen Joint Undertaking, 2018 ANNUAL WORK PLAN and BUDGET, p.68 http://www.fch.europa.eu/sites/default/files/FCH2JU2018AW\%WPand\%20Budget

[7] Le Jin, Wanbing Guan, Xiao Ma, Huijuan Zhai, Wei Guo Wang, Journal of Power Sources, 253 (2014) 305-314

[8] Y. Zhu and K.Tomsovic, Electric Power Systems Research 62 (2002) 1-11

[9] Z. Stoynov, D. Vladikova, B. Burdin, „Recommendation for Measurements of Volt Ampere Characteristics of Fuel Cells for Diagnostic Purposes“, Handbook of Test Procedures and Protocols, Chapter 3: ENDURANCE Procedures and Protocols, http://www.durablepower.eu/index.php/handbook

[10] http://www.durablepower.eu

[11] http://www.sophia-project.eu

[12] J. Laurencin, M. Hubert, D. Ferreira Sanchez, S. Pylypko, M. Morales, A. Morata, B. Morel, D. Montinaro, F. Lefebvre-Joud, E. Siebert, Electrochimica acta, 241 (2017) $459-476$.

[13] T.L. Skafte, J. Hjelm, P. Blennow, C. Graves, "Quantitative review of degradation and lifetime of solid oxide cells and stacks", in: 12th European SOFC \& SOE Forum, B0501, Lucerne, Switzerland, 2016.

[14] J. Schefold, A. Brisse, F. Tietz, J. Electrochem. Soc., 159(2) (2012) A137-A144.

[15] R. Kiebach, W.-W. Zhang, W. Zhang, M. Chen, K. Norrman, H.-J. Wang, J.R. Bowen, R. Barfod, P.V. Hendriksen, J. Power Sources, 283 (2015) 151-161.

[16] A. Brisse, J. Schefold, M. Zahid, Int. J. Hydrogen Energy, 33(20) (2008) 5375-5382. 
[17] H. Orui, K. Nozawa, K. Watanabe, S. Sugita, R. Chiba, T. Komatsu, H. Arai, M. Arakawa, J. Electrochem. Soc., 155(11) (2008) B1110-B1116.

[18] F. Tietz, V. A. C. Haanappel, A. Mai, J. Mertens, D. Stöver, J. Power Sources 156(1) (2006) 20-22. 
TABLES

Table 1. Tested cells, corresponding testing conditions and calculated Degradation Rates

\begin{tabular}{llll}
\hline & Cell C1 & Cell C2 & Cell C3 \\
\hline Temperature [ $\left.{ }^{\circ} \mathrm{C}\right]$ & 750 & 850 & 850 \\
Mode & Fuel Cell & Fuel Cell & Electrolysis \\
$\mathrm{H}_{2} / \mathrm{H}_{2} \mathrm{O}$ ratio & $100 / 0$ & $50 / 50$ & $50 / 50$ \\
Fuel/Steam utilization [\%] & 60 & 60 & 60 \\
Test duration [hrs] & 9000 & 1000 & 1000 \\
Current density [A.cm $\left.{ }^{-2}\right]$ & +0.50 & +0.50 & -0.50 \\
$\mathrm{DR}_{\mathrm{U}}\left[\mathrm{mV} \cdot \mathrm{kh}^{-1}\right]$ & (I) 13.5 (II) 4.1 & 13.0 & 42.0 \\
$\mathrm{DR}_{\mathrm{U}}\left[\% \cdot \mathrm{kh}^{-1}\right]$ & (I) 1.6 (II) 0.5 & 1.6 & 3.9 \\
$\mathrm{DR}_{\mathrm{Rd}, \mathrm{min}}\left[{\left.\mathrm{m} \Omega . \mathrm{kh}^{-1}\right]}^{-1}\right.$ & (I) 17.6 (II) 6.2 & 38.0 & 71.0 \\
$\mathrm{DR}_{\mathrm{Rd}, \mathrm{min}}\left[\% \cdot \mathrm{kh}^{-1}\right]$ & (I) 4.4 (II) 1.3 & 17.1 & 34.8 \\
$\mathrm{DR}_{\Delta \mathrm{U}^{*}\left[\mathrm{mV} . \mathrm{kh}^{-1}\right]}$ & (I) 6.6 (II) 1.6 & - & - \\
$\mathrm{DR}_{\Delta \mathrm{U}^{*}\left[\% . \mathrm{kh}^{-1}\right]}$ & (I) 5.6 (II) 1.3 & - & - \\
\hline
\end{tabular}




\section{FIGURE CAPTIONS}

Fig. 1. Current-voltage and current-power curves (schematic presentation)

Fig. 2. DiVA procedure: (a) i-V curve and $R_{\mathrm{d}} / I$ dependence; (b) spectral transform of the $R_{\mathrm{d}} / I$ dependence; (c) twin spectra; (d) determination of the performance indicator $\Delta U^{*}$ (schematic presentations)

Fig. 3. DRA of i-V curves measured on 4-cells stack before and after the first thermal cycle: (a) i-V curves; (b) spectral presentation of the $R_{\mathrm{d}} / I$ dependence; (c) performance histogram in respect to $R_{\mathrm{d}, \min }, \Delta U^{*}$ and $P_{\max }$ changes (in \%)

Fig. 4. DRA of cell $\mathrm{C} 1$ for testing time 9000 hours: (a) i-V curves; (b) $R_{\mathrm{d}, \min }$ and $\Delta U^{*}$ change during testing; (c) impedance diagrams for selected testing time

Fig. 5. i-V curves for cells C2 and C3 before and after 1000 hours of operation 


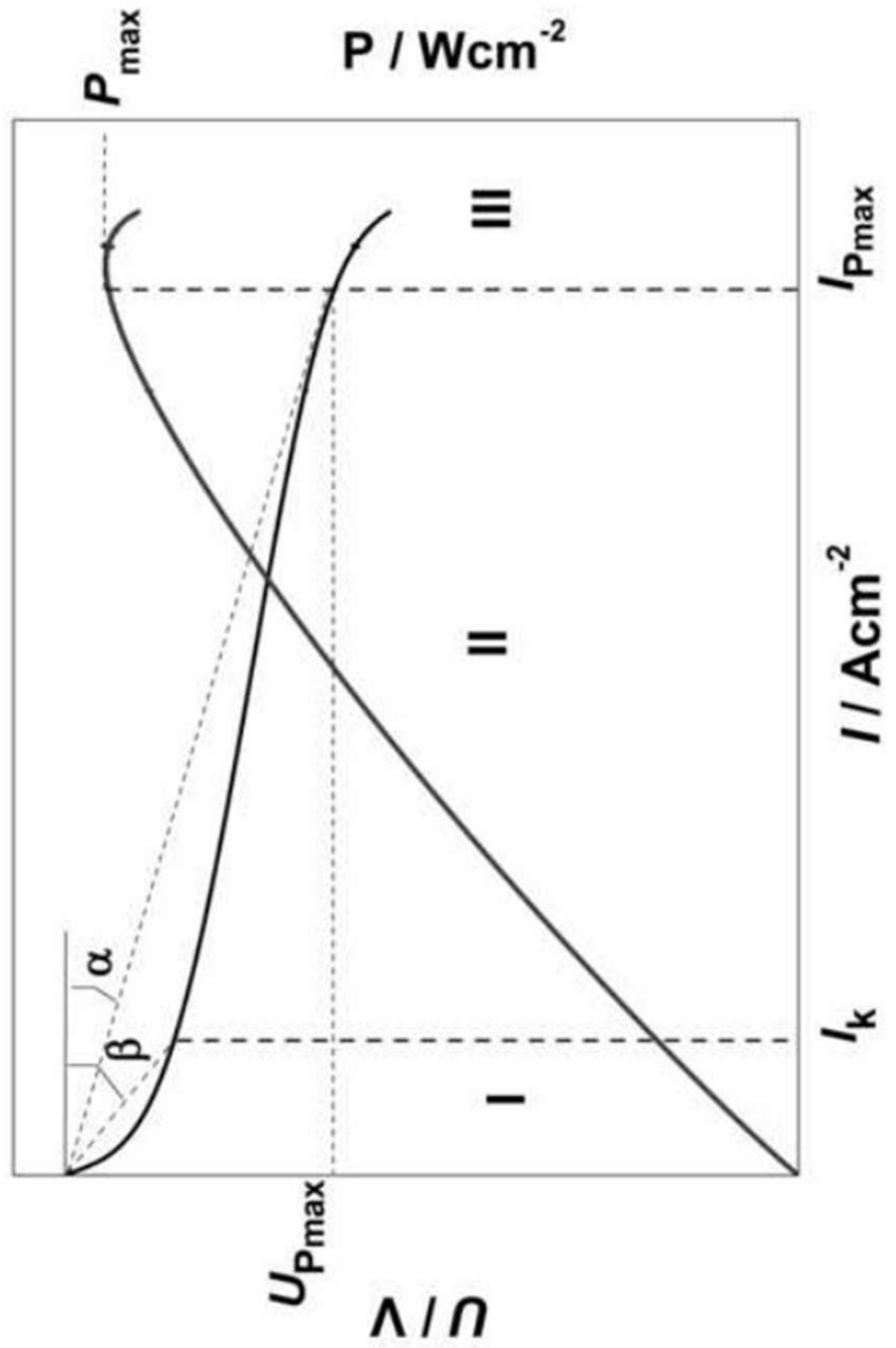

Fig. 1 


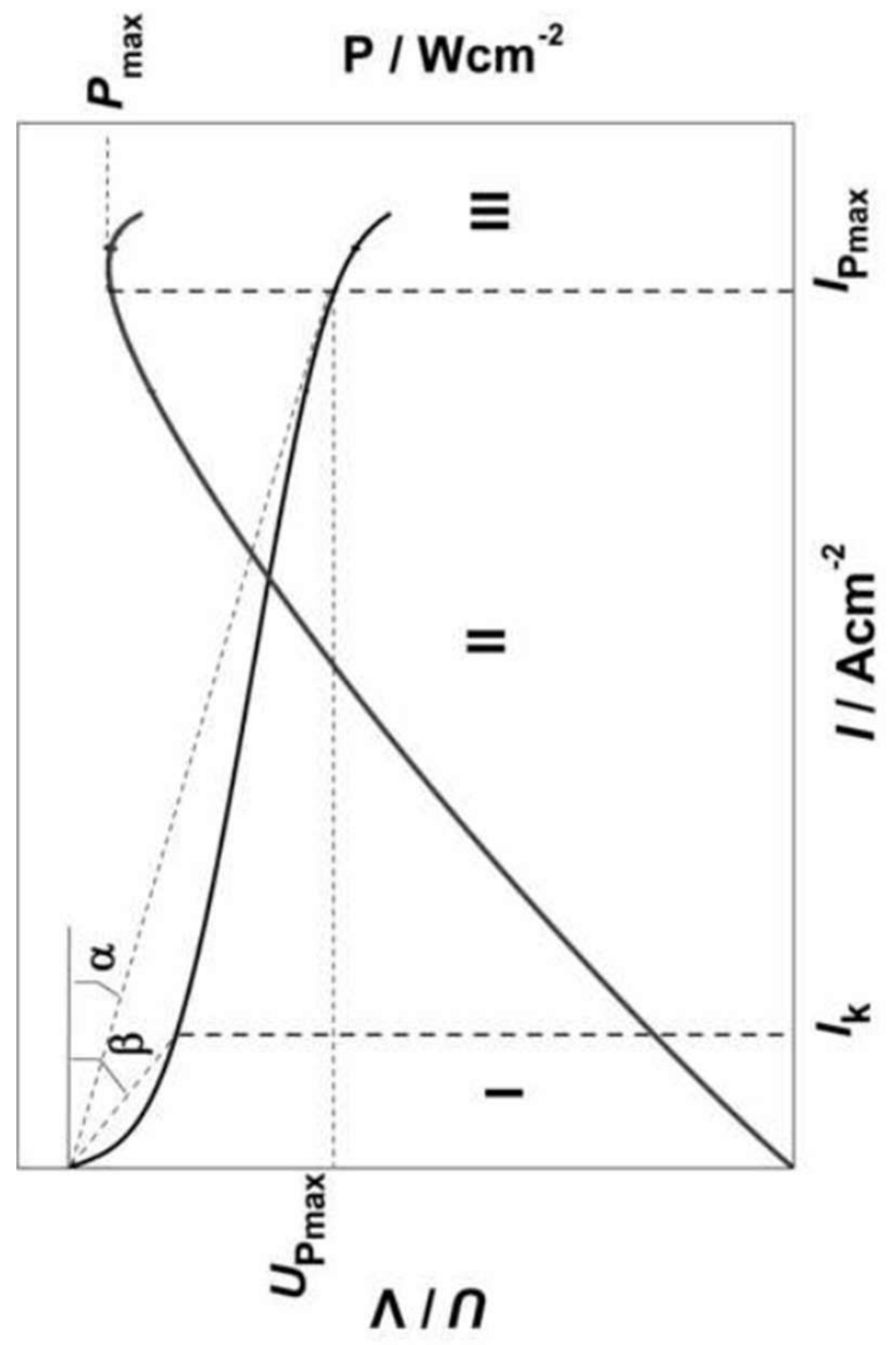

Fig. 2a 


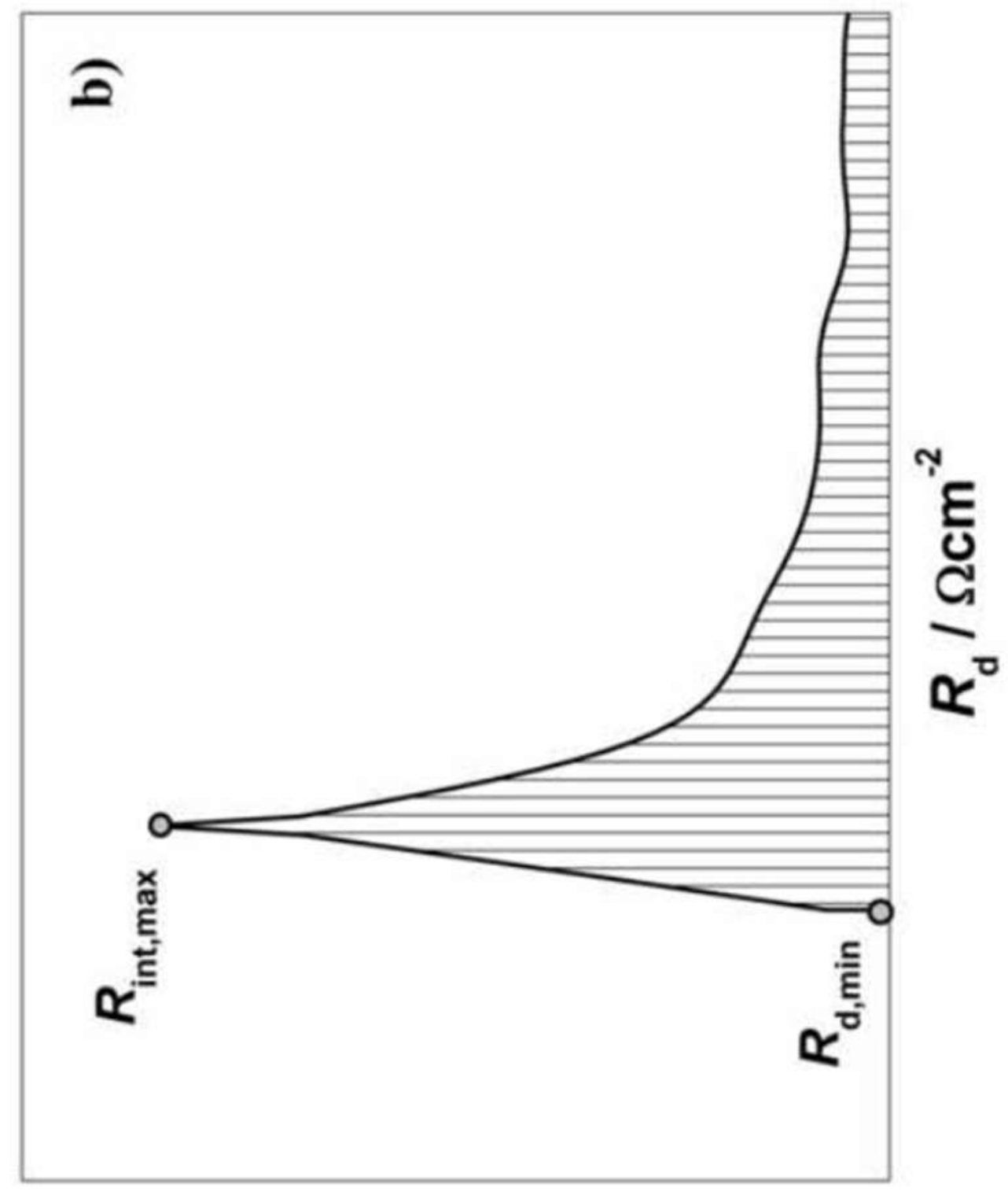

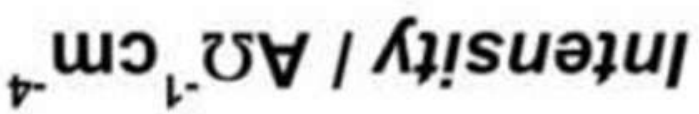

Fig. 2b 


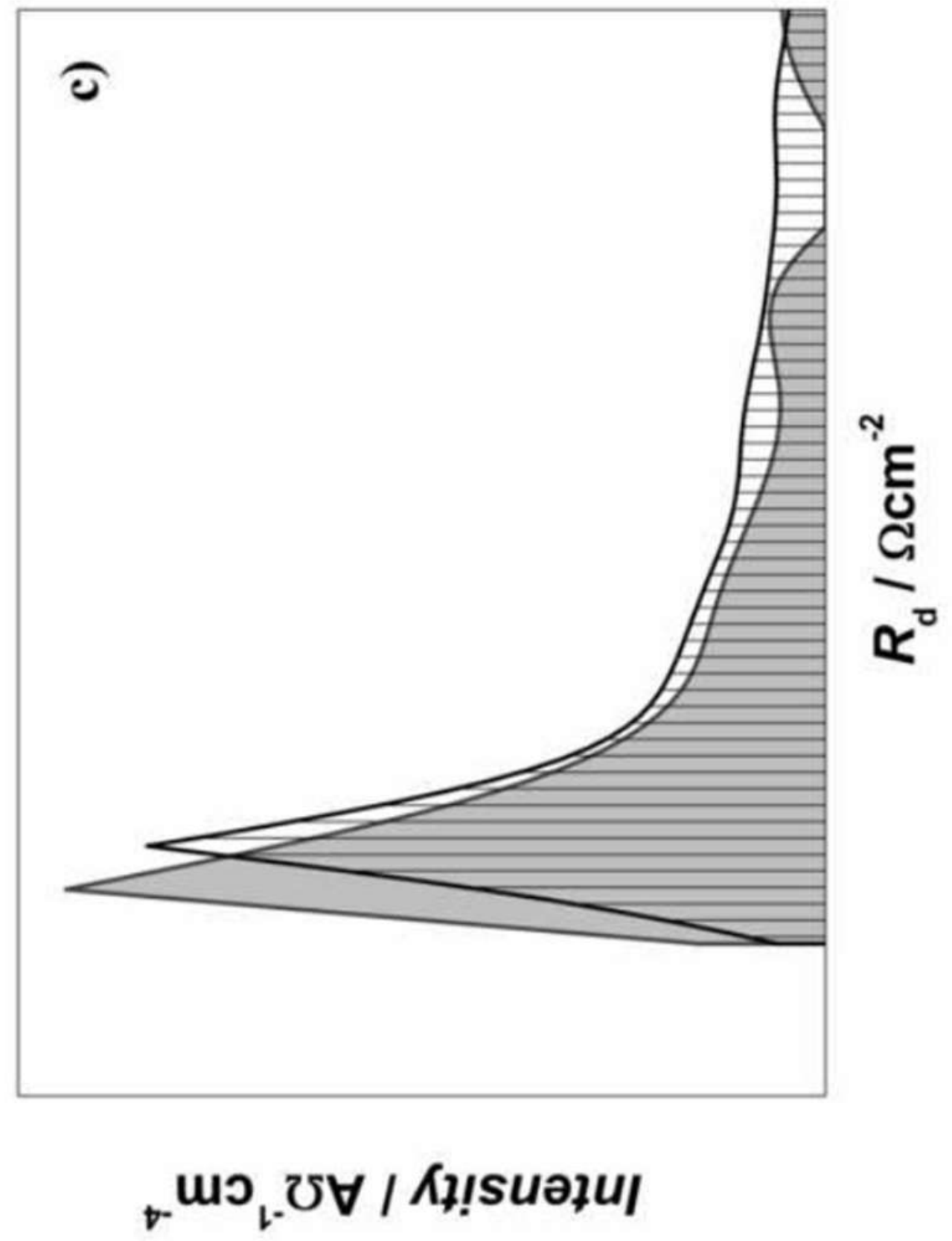

Fig. 2c 


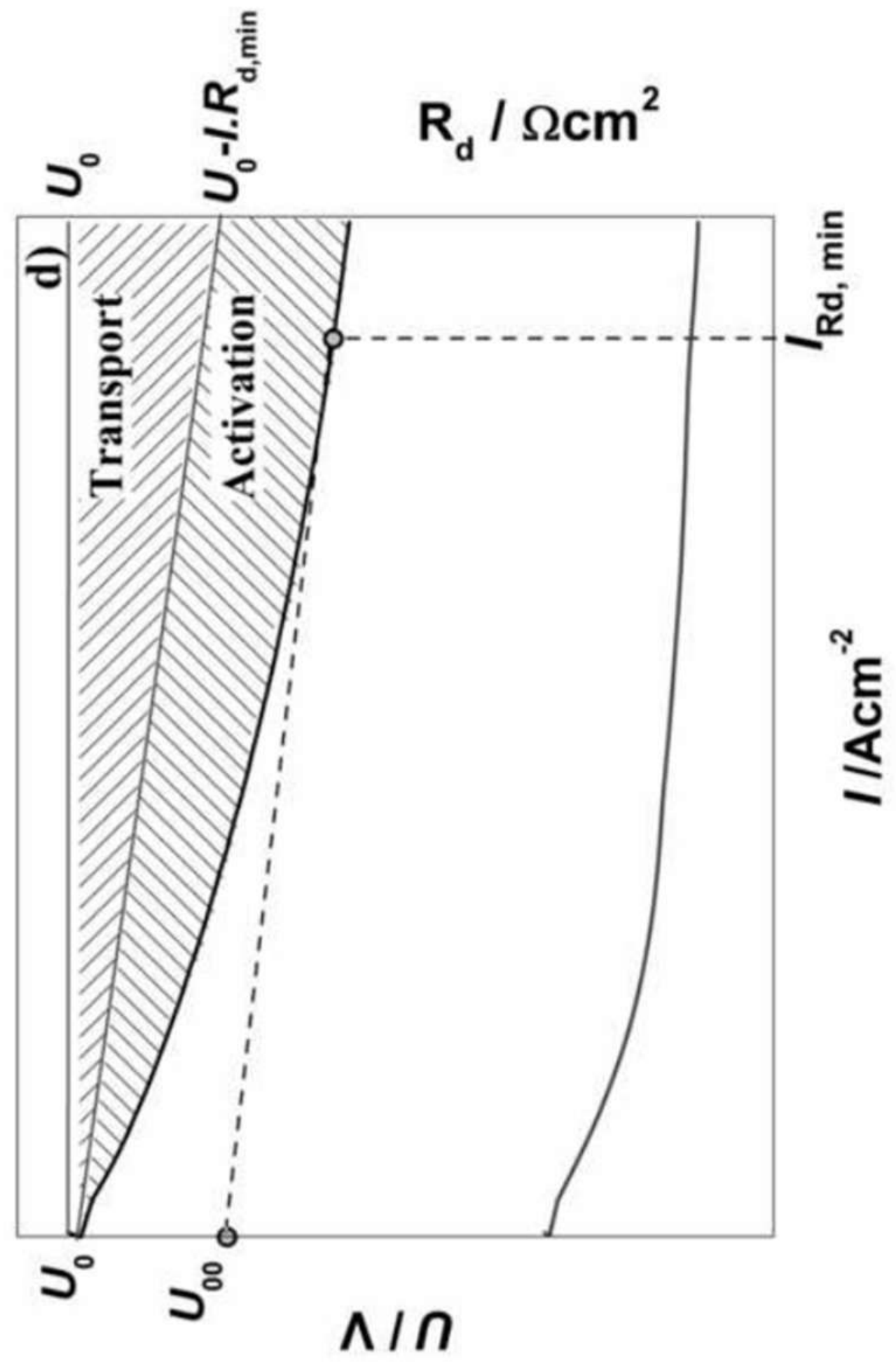

Fig. 2d 


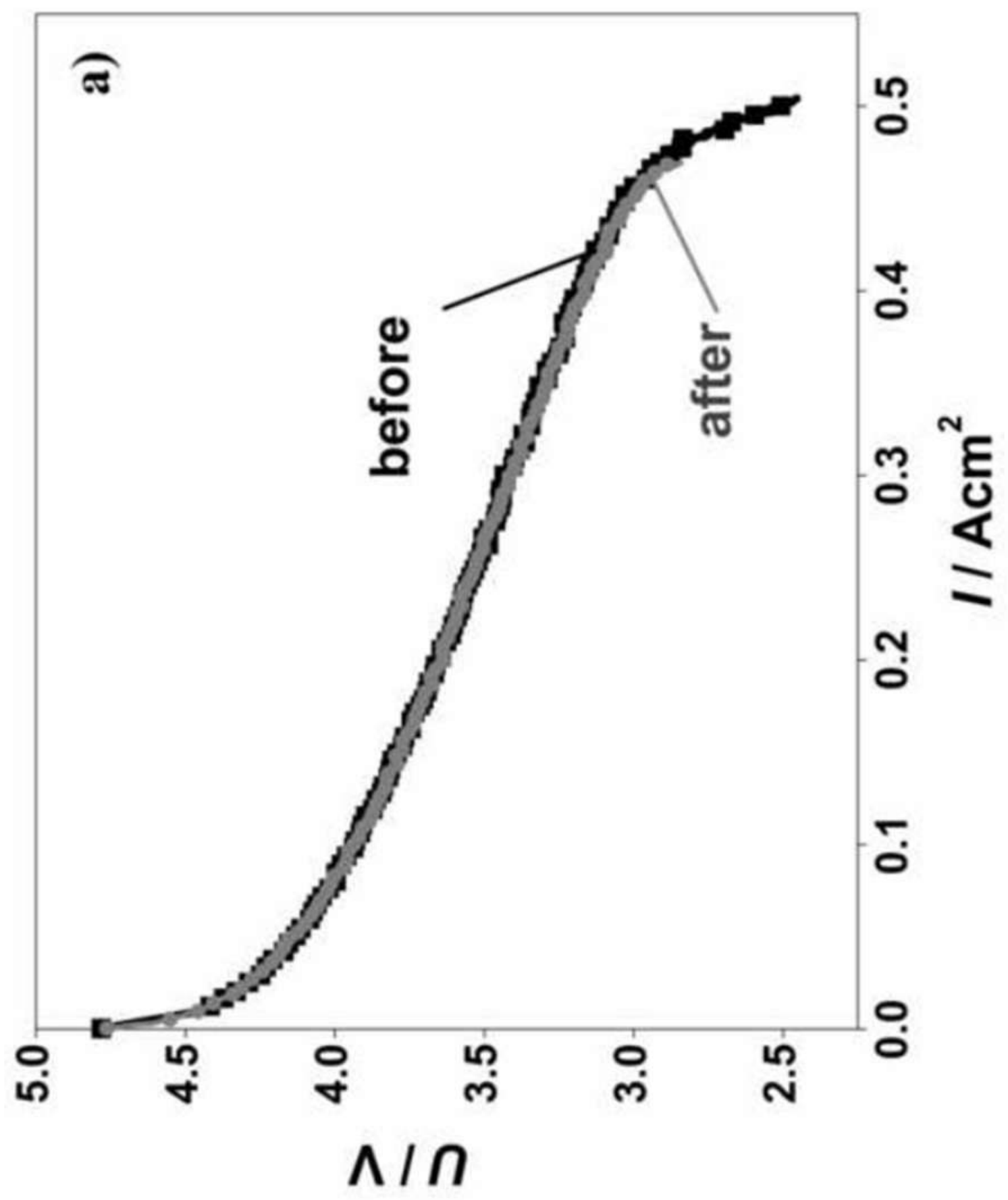

Fig. 3a 


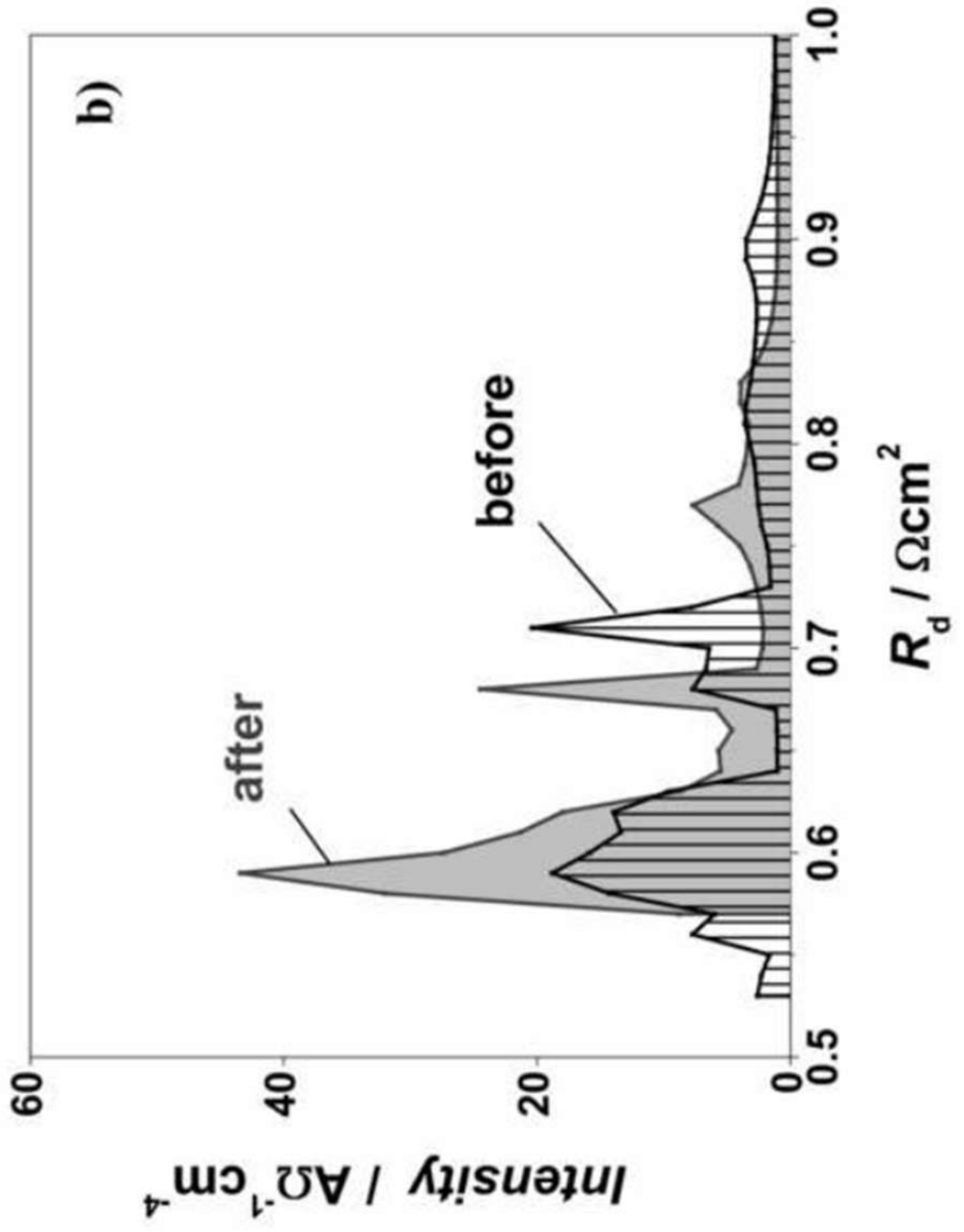

Fig. 3b 


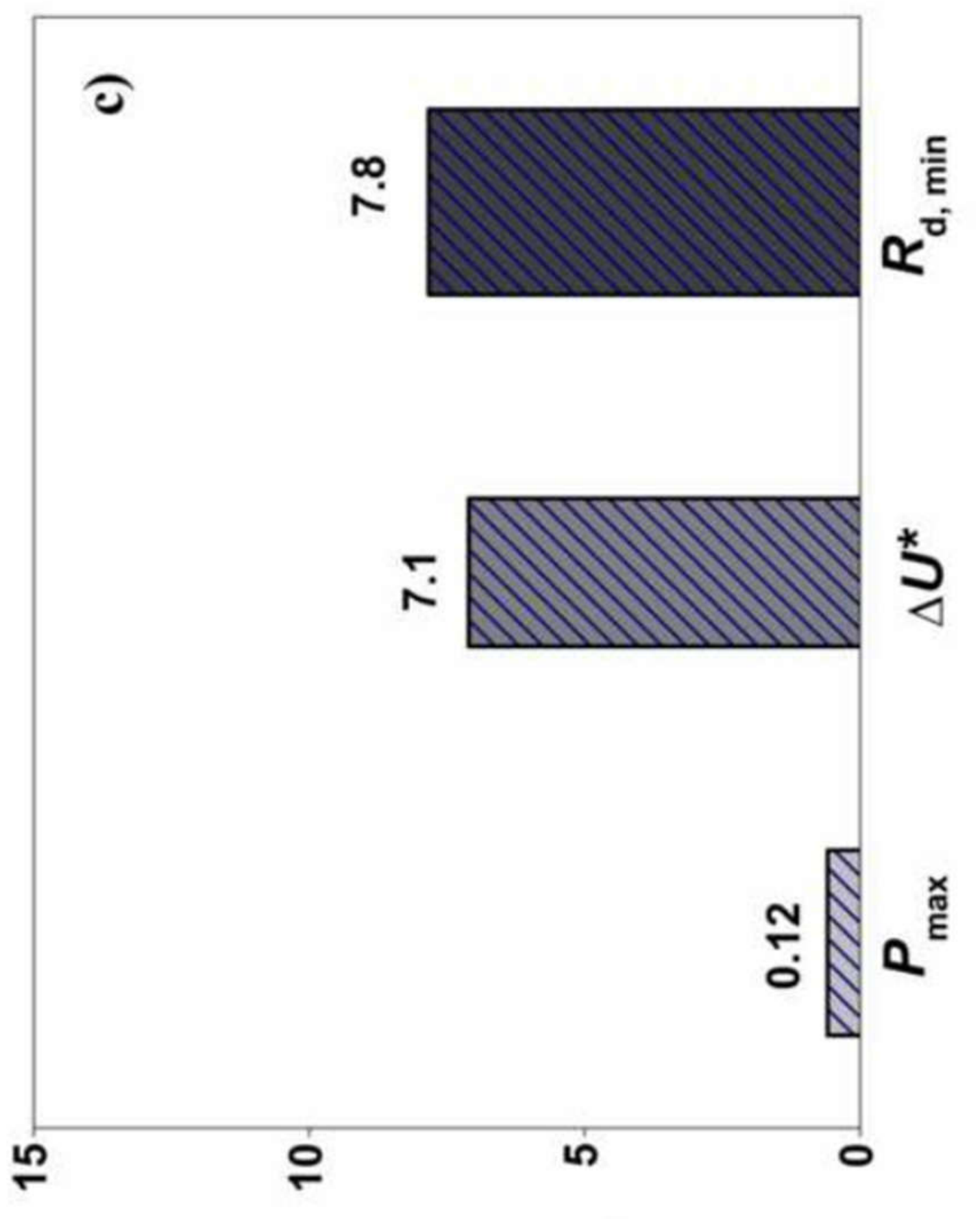

\% / әуеу иопередбәа

Fig. 3c 


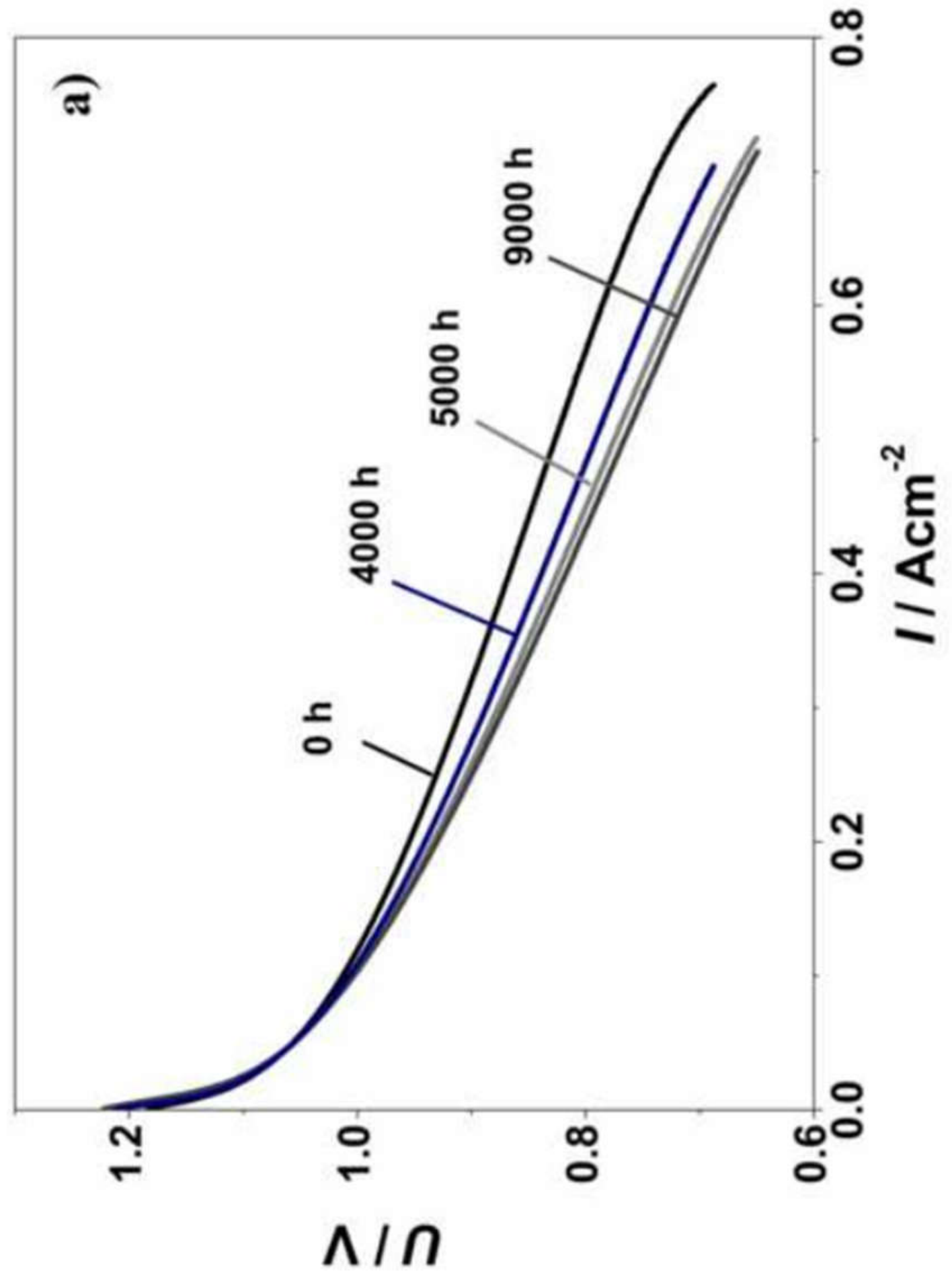

Fig. 4a 


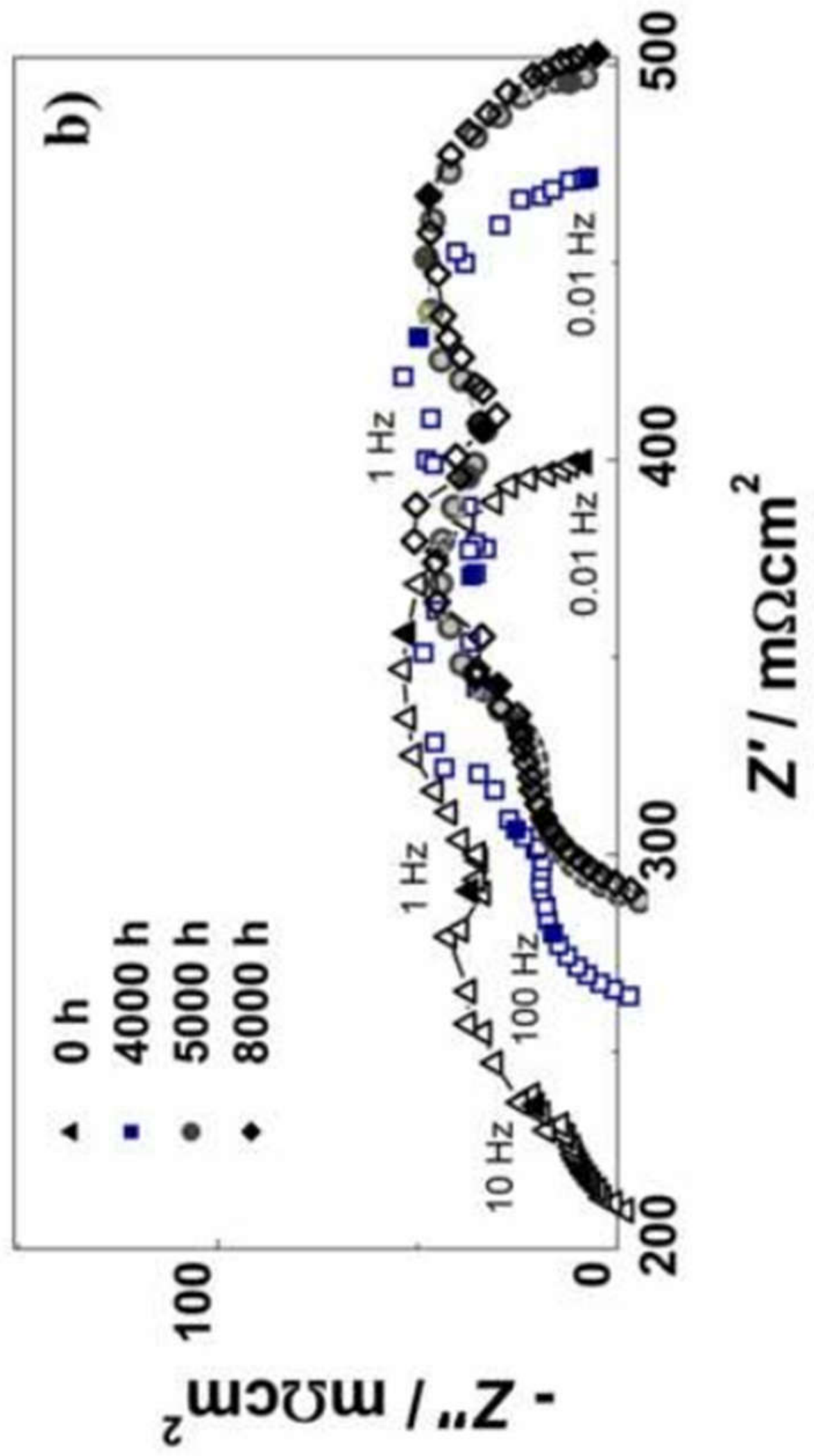

Fig. 4b 


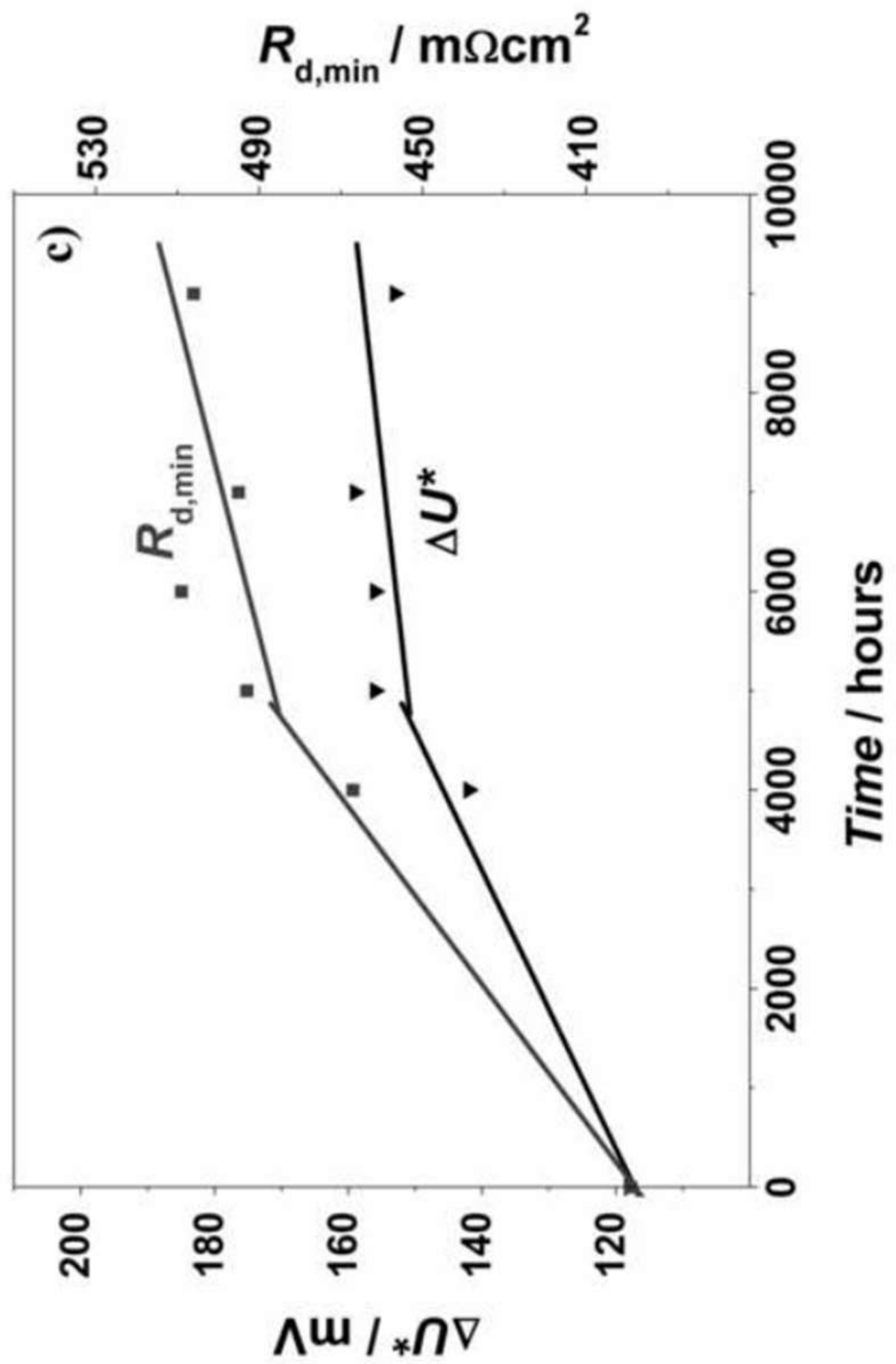

Fig. 4c 


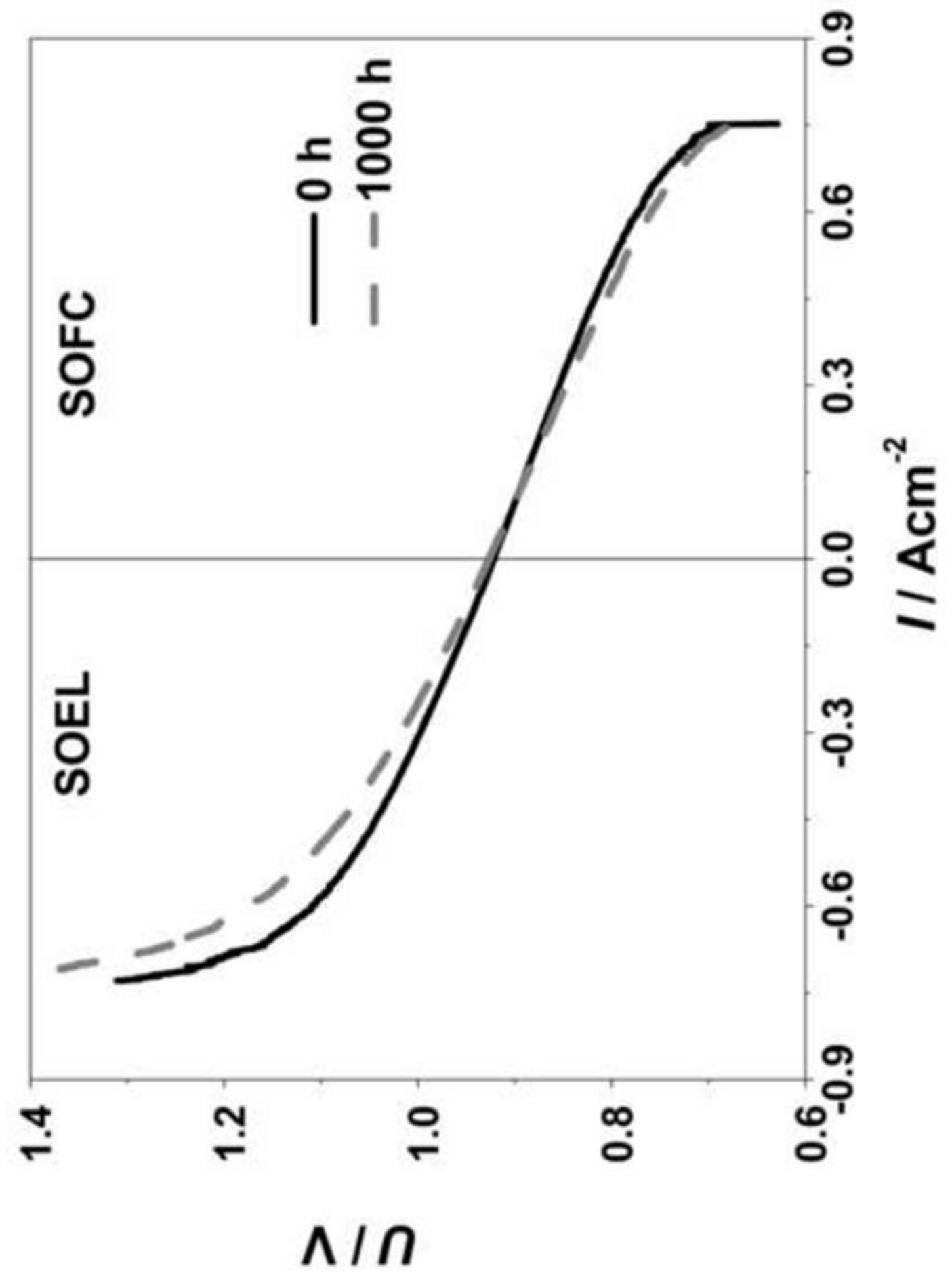

Fig. 5 